\title{
Effect of Helicobacter pylori infection on malignancy of undifferentiated-type gastric cancer
}

\author{
Masami Tanaka* (10, Shu Hoteya, Daisuke Kikuchi, Kosuke Nomura, Yorinari Ochiai, Takayuki Okamura, \\ Junnosuke Hayasaka, Yugo Suzuki, Yutaka Mitsunaga, Nobuhiro Dan, Hiroyuki Odagiri, Satoshi Yamashita and \\ Akira Matsui
}

\begin{abstract}
Background: Although almost all cases of gastric cancer are caused by Helicobacter pylori (HP) infection, there are some rare exceptions. Furthermore, the clinicopathological characteristics of gastric cancer may differ depending on HP infection status. This study aimed to determine the clinicopathological characteristics of undifferentiated-type gastric cancer (UD-GC) according to HP status.

Methods: The study involved 83 patients with UD-GC who were selected from 1559 patients with gastric cancer who underwent endoscopic resection at our hospital and whose HP infection status was confirmed. Clinicopathological characteristics were evaluated according to HP status (eradicated, $n=28$; infected, $n=32$; not infected, $n=23$ ).

Results: In patients without HP infection, UD-GCs were $<20 \mathrm{~mm}$ and intramucosal with no vascular invasion. In patients with eradicated HP, there was no correlation between development of UD-GC and time since eradication. Nine of twelve patients with a tumor detected $\geq 5$ years after eradication had undergone yearly endoscopy. Submucosal invasion was observed in two of four patients and lymphovascular invasion in three of four patients whose UD-GC was detected $\geq 10$ years after eradication. There was no significant between-group difference in the frequency of lesions with invasion into the submucosal layer or deeper (14.3\%, 10.5\%, and 0\% in the UD-E, UD-I, and UD-U groups, respectively).

Conclusion: The clinicopathological characteristics of UD-GC were similar between HP-infected patients and HPeradicated patients. Three of four patients with eradicated HP whose UD-GC developed $\geq 10$ years after eradication were not eligible for endoscopic treatment and required additional surgery resection. In contrast, UD-GC was curable by endoscopic resection in all patients without HP infection.
\end{abstract}

Keywords: Helicobacter pylori, Undifferentiated carcinoma, Gastric cancer, Malignancy

\section{Background}

Almost all cases of gastric cancer are caused by Helicobacter pylori (HP) infection [1-3]. Although rare, gastric cancer can also develop in the absence of HP infection

*Correspondence: m-tanaka@toranomon.gr.jp

Department of Gastroenterology, Toranomon Hospital, 2-2-2 Toranomon,

Minato-ku, Tokyo 105-8470, Japan
[4-6], and the characteristics of gastric cancer without HP infection have become clearer with accumulation of cases [7-10]. The clinicopathological features of gastric cancer differ according to HP infection status. Differentiated-type gastric cancer (D-GC) undergoes morphological changes upon eradication of HP [11-13], and changes on the mucosal surface of the tumor to a non-cancerous appearance after eradication hinder endoscopic diagnosis 
[14]. Meanwhile, the degree of malignancy of undifferentiated-type gastric cancer (UD-GC), including proliferative ability and progression rate, is reported to be higher in patients with HP infection (both post-eradication and current infection cases) than in those without HP infection [15]. However, details of UD-GC post-eradication remain unclear. The latest endoscopic mucosal resection/endoscopic submucosal dissection guidelines state that intramucosal lesions measuring $\leq 20 \mathrm{~mm}$ and ulcernegative UD-GC are absolute indications for endoscopic resection [16]. Therefore, it is important for endoscopists diagnosing and treating UD-GC to have a good knowledge of the clinicopathological features of this tumor according to HP infection status.

In this study, we classified patients with UD-GC according to HP status as a post-eradication (UD-E) group, a current infection (UD-I) group, and a noninfected (UD-U) group with the aim of clarifying the clinicopathological features of each group.

\section{Methods}

The study involved patients with gastric cancer who underwent endoscopic resection at Toranomon Hospital between June 2011 and December 2019. A flow chart showing the patient selection process is provided in Fig. 1. After excluding 108 cases with residual gastric cancer and 446 with unknown HP infection status or unclear time of eradication, we reviewed 1559 cases (2032 lesions) with confirmed HP status. This study was approved by the ethics committee of Toranomon Hospital, approval number 1173 .

HP eradication group was defined as follows: known time of eradication and HP-negative status confirmed by a stool antigen test (Meridian Inc., Cincinnati, $\mathrm{OH}$ ) or a ${ }^{13} \mathrm{C}$-urea breath test (Otsuka Pharmaceutical Co., Ltd., Tokushima, Japan). Current HP infection was defined as a positive urea breath test or stool antigen test, or a positive serum antibody test (E-plate test; Eiken Chemical Co., Ltd., Tokyo, Japan) plus endoscopic findings suggesting current HP infection [17]. Patients were deemed not to have HP infection if the following four criteria were satisfied: no history of HP eradication; a negative serum antibody test $(<3 \mathrm{U} /$ $\mathrm{mL}$ ) and a negative stool antigen test or urea breath test; no atrophy of the background mucosa on pathological examination of the specimen obtained during endoscopic resection; and no atrophy according to the Kimura-Takemoto classification [18] with regular arrangement of the collecting venules in the lesser curvature of the gastric angle [19].

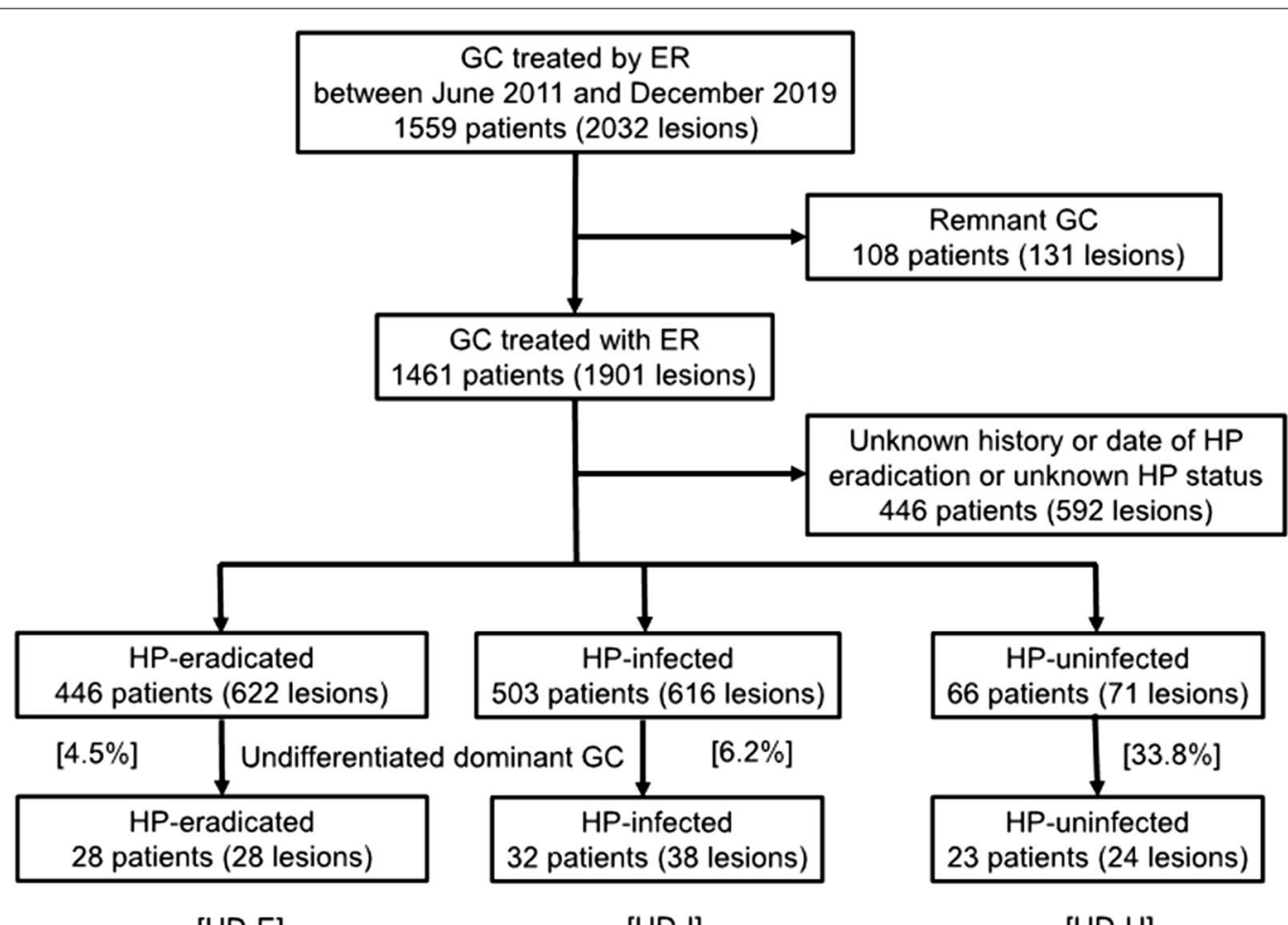

[UD-E]

[UD-I]

[UD-U]

Fig. 1 Flow chart showing the patient selection process. Undifferentiated-type cases with confirmed HP infection status were extracted for analysis. GC, gastric cancer; ER, endoscopic resection; HP, Helicobacter pylori; UD-E, post-eradication group: UD-I, current infection group; UD-U, uninfected group 
HP status was confirmed in 446 cases (622 lesions) in HP-eradicated, 503 cases (616 lesions) in HP-infected, and 66 cases (71 lesions) in HP-uninfected. UD-GC cases were then extracted for analysis; there were 28 cases (28 lesions) in the UD-E group, 32 cases (38 lesions) in the UD-I group, and 23 cases (24 lesions) in the UD-U group.

The following clinicopathological characteristics were evaluated: patient demographics (age and sex), endoscopic findings (degree of atrophy, macroscopic type, site, and color tone), and histopathological features (findings, tumor size, depth of invasion, and lymphovascular invasion). In the UD-E group, we also evaluated the time from eradication, tumor size, color tone, depth of invasion, and lymphovascular invasion. The Kimura-Takemoto classification [18] was used to evaluate the degree of atrophy (Close[C]-0, none; $\mathrm{C}-1$ and $\mathrm{C}-2$, mild; C-3 to Open[O]-3), moderate-severe). Color tone was classified as discolored or reddish; if mixed, the predominant tone was selected. Histopathological findings were classified into UD-GC or D-GC; when both types were present, the predominant type was used. UD-GC cases were further divided into pure signet ring cell carcinoma or other type (poorly differentiated adenocarcinoma or mixed poorly differentiated adenocarcinoma and signet ring cell carcinoma) [20].

The clinicopathological features were compared between the three groups using chi square test, Fisher exact test, Kruskal Wallis test, and Scheffe test. All statistical analyses were performed using SPSS version 27 (IBM Corp., Armonk, NY). A $p$-value $<0.05$ was considered statistically significant.

\section{Results}

The ratio of UD-GC to all tumors in each HP infection status group was significantly higher in the UD-U group $(33.8 \%)$ than in the UD-E and UD-I groups $(4.5 \%$ and $6.2 \%$, respectively), which is shown in Fig. 1.

\section{Patient characteristics}

The characteristics of the patients in the UD-E, UD-I, and UD-U groups are shown in Table 1 . The male proportion was $64.3 \%$ in the UD-E group, $43.8 \%$ in the UD-I group, and $87.0 \%$ in the UD-U group. There was a significantly higher proportion of women in the UD-I group than in the UD-U group ( $p=0.001$, UD-I vs UD-U; $p=0.105$, UD-U vs UD-E). However, there was no significant difference in the sex distribution between the UD-E and UD-I groups or between the UD-E and UD-U groups. The mean age \pm standard deviation was $63.1 \pm 14.3$, $64.0 \pm 13.4$, and $56.3 \pm 9.15$ years, respectively, in the UD-E, UD-I, and UD-U groups; patients in the UD-U group were significantly younger than those in the UD-E and UD-I groups ( $p=0.017$ and $p=0.019$, respectively). The background mucosa was significantly more atrophied in the UD-E and UD-I groups (both $p<0.01$ ) than in the UD-U group (which had no cases of atrophy).

\section{Endoscopic findings}

Endoscopic findings are shown in Table 2. The lesions were located in the upper third, middle third, and lower third of the stomach in $2 / 9 / 17$ cases in the UD-E group, $2 / 22 / 14$ cases in the UD-I group, and $1 / 5 / 18$ cases in the UD-U group, indicating that the middle third of the stomach was a significantly more common site in the

Table 1 Patient characteristics in the group with undifferentiated-type gastric cancer according to Helicobacter pylori infection status

\begin{tabular}{|c|c|c|c|c|}
\hline & UD-E (A) & UD-I (B) & UD-U (C) & Significance \\
\hline Patients (lesions) with undifferentiated AC, n & $28(28)$ & $32(38)$ & $23(24)$ & \\
\hline Sex & & & & $\begin{array}{l}p=0.001(A \text { vs } B \text { vs } C) \\
p=0.001(B \text { vs } C) \\
\text { NS (A vs B) } \\
\text { NS (C vs } A)\end{array}$ \\
\hline Male & $18(64.3 \%)$ & $14(43.8 \%)$ & $20(87.0 \%)$ & \\
\hline Female & $10(35.7 \%)$ & $18(56.2 \%)$ & $3(23.0 \%)$ & \\
\hline Age, years (mean $\pm S D$ ) & $63.1 \pm 14.3$ & $64.0 \pm 13.4$ & $56.3 \pm 9.15$ & $\begin{array}{l}p=0.026(A \text { vs B vs } C) \\
p=N . S(A \text { vs B }) \\
p=0.0012(B \text { vs } C) \\
p=0.008(C \text { vs } A)\end{array}$ \\
\hline Atrophy & & & & $\begin{array}{l}p<0.01(A \text { vs } B \text { vs } C) \\
p<0.01(B \text { vs } C) \\
p<0.01(C \text { vs } A) \\
p=0.101(A \text { vs } B)\end{array}$ \\
\hline None to Mild(C-0-C-2) & $7(25.0 \%)$ & $3(9.4 \%)$ & $23(100 \%)$ & \\
\hline Moderate to Severe $(\mathrm{C}-3-\mathrm{O} 3)$ & $21(75.0 \%)$ & $29(90.6 \%)$ & $0(0 \%)$ & \\
\hline
\end{tabular}

AC, adenocarcinoma; NS, not statistically significant; SD, standard deviation; UD-E, post-eradication group: UD-I, current infection group; UD-U, noninfected group 
Table 2 Endoscopic findings in patients with undifferentiated-type gastric cancer according to Helicobacter pylori infection status

\begin{tabular}{|c|c|c|c|c|}
\hline & UD-E (A) & UD-I (B) & UD-U (C) & Significance \\
\hline Location & & & & $\begin{array}{l}p=0.048(A \text { vs B vs } C) \\
p=0.002(B \text { vs } C) \\
\text { NS (A vs B, C vs A) }\end{array}$ \\
\hline Upper third & $2(7.1 \%)$ & $2(5.3 \%)$ & $1(4.2 \%)$ & \\
\hline Middle third & $9(32.1 \%)$ & $22(57.9 \%)$ & $5(20.8 \%)$ & \\
\hline Lower third & $17(60.8 \%)$ & $14(36.8 \%)$ & $18(75.0 \%)$ & \\
\hline Macroscopic appearance & & & & $\begin{array}{l}\text { NS ( } A \text { vs } B \text { vs } C) \\
N S(A \vee s, B, B \text { vs } C, C \text { vs } A)\end{array}$ \\
\hline Elevated & $1(3.6 \%)$ & $2(5.3 \%)$ & $0(0 \%)$ & \\
\hline Flat or depressed & $27(96.4 \%)$ & $36(94.7 \%)$ & $24(100 \%)$ & \\
\hline Color tone & & & & $\begin{array}{l}p<0.001 \text { (A vs B vs } C) \\
p<0.001(B \text { vs } C, C \text { vs } A) \\
\text { NS (A vs B) }\end{array}$ \\
\hline Discolored dominant & $16(57.1 \%)$ & $16(42.1 \%)$ & $24(100 \%)$ & \\
\hline Reddish dominant & $12(42.9 \%)$ & $22(57.9 \%)$ & $0(0 \%)$ & \\
\hline
\end{tabular}

NS, not significant; UD-E, post-eradication group: UD-I, current infection group; UD-U, non-infected group

UD-I group than in the UD-U group $(p=0.002)$. There was no significant difference in macroscopic type of the lesions between the groups. The color tone was reddish in about half of the cases in the UD-E and UD-I groups ( $42.9 \%$ and $57.9 \%$, respectively), whereas all cases in the UD-U group were discolored $(p<0.001$, UD-I vs UD-U; $p<0.001$, UD-U vs UD-E).

\section{Histopathological features}

Mean tumor diameter was significantly smaller in the UD-U group $(10.1 \pm 5.4 \mathrm{~mm})$ than in the UD-E group $(19.4 \pm 11.7 \mathrm{~mm} ; p=0.024)$ and UD-I group $(18.6 \pm 10.2 \mathrm{~mm} ; p=0.028)$. In the UD-U group, the majority of lesions $(87.5 \%, 21 / 24)$ were pure signet ring carcinoma; this type of gastric cancer was significantly less common in the UD-E and UD-I groups $(50.0 \%$ and $28.9 \%$, respectively; both $p<0.01$; Table 3 ).
There was no significant between-group difference in the frequency of lesions with invasion into the submucosal layer or deeper $(14.3 \%, 10.5 \%$, and $0 \%$ in the UD-E, UD-I, and UD-U groups, respectively). All lesions in the UD-U group were intramucosal tumors. Lymphovascular invasion was seen only in the UD-E group (3 cases, $10.7 \%)$. Two of the 3 lesions with lymphovascular invasion had a tumor diameter of $\geq 30 \mathrm{~mm}$. There was no significant difference compared either with the UD-I or UD-I group.

\section{Relationship of time since eradication with tumor diameter, color tone, and depth of invasion in the UD-E group}

Figure 2 shows the relationship of time since eradication with tumor diameter, color tone, and depth of invasion. The median interval between eradication and detection of gastric cancer was 56 (9-240) months in the UD-E group.

Table 3 Histopathological findings in the patients with undifferentiated gastric cancer according to Helicobacter pylori infection status

\begin{tabular}{|c|c|c|c|c|}
\hline & UD-E (A) & UD-I (B) & UD-U (C) & Significance \\
\hline Maximum lesion diameter, mean \pm SD & $19.3 \pm 11.7$ & $18.6 \pm 10.2$ & $10.1 \pm 5.4$ & $\begin{array}{l}P<0.01(A \text { vs } B \text { vs } C) \\
p=0.001(C \text { vs } A) \\
p=0.005(B \text { vs } C) \\
\text { NS (A vs B) }\end{array}$ \\
\hline Pathological type, $\%$ of pure signet ring cell CA & $50.0 \%(14 / 28)$ & $28.9 \%(11 / 38)$ & $87.5 \%(21 / 24)$ & $\begin{array}{l}p<0.001(A \text { vs } B \text { vs } C) \\
p<0.001(B \text { vs } C, C \text { vs } A) \\
\text { NS (A vs } B)\end{array}$ \\
\hline Depth, \% of SM invasive cancer & $14.3 \%(4 / 28)$ & $10.5 \%(4 / 38)$ & $0 \%(0 / 24)$ & $\begin{array}{l}N S(A \text { vs } B \text { vs } C) \\
N S(A \vee s, B, B \text { vs } C, C \text { vs } A)\end{array}$ \\
\hline Lymphovascular invasion, \% of undifferentiated CA & $10.7 \%(3 / 28)$ & $0 \%(0 / 38)$ & $0 \%(0 / 24)$ & $\begin{array}{l}\text { NS (A vs } B \text { vs } C) \\
N S(A \text { vs } B, B \text { vs } C, C \text { vs } A)\end{array}$ \\
\hline
\end{tabular}

CA, carcinoma; NS, not statistically significant; SD, standard deviation; SM, submucosal; UD-E, post-eradication group: UD-I, current infection group; UD-U, noninfected group 


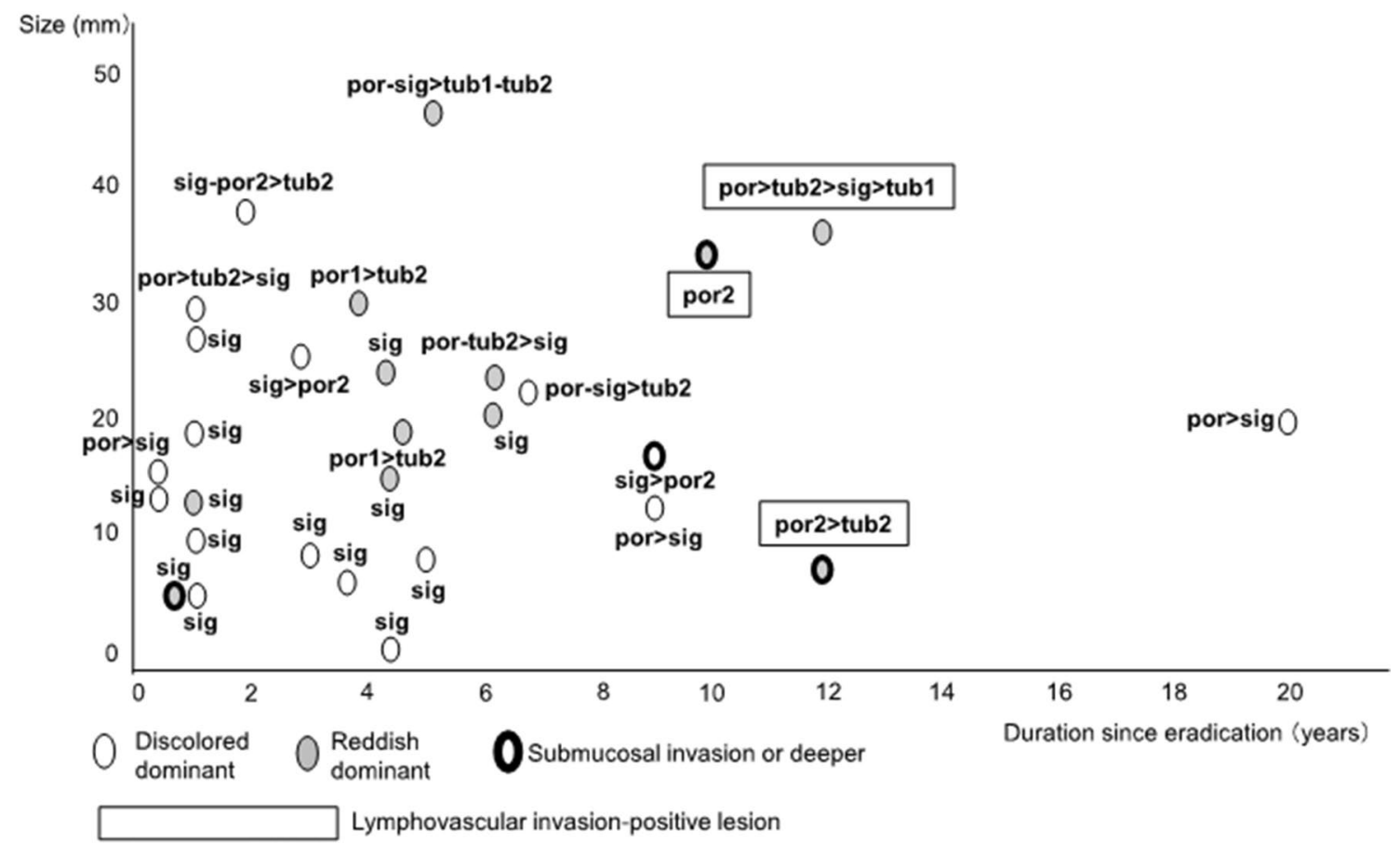

Fig. 2 Relationship of time since eradication with tumor diameter, color tone, lymphovascular invasion, and depth of invasion in the post-eradication UD-GC cases. Por, poorly differentiated adenocarcinoma; sig, signet ring cell carcinoma; tub, tubular adenocarcinoma; UD-GC, undifferentiated-type gastric cancer

About $60 \%$ of UD-GCs (17 of 28 lesions) are detected within 5 years. Pure signet-ring cell adenocarcinomas are often detected early after HP eradication and tends to be smaller in size than the pathologically pure-poorly differentiated UD-GC and mixed UD-GCs. UD-GC was detected $\geq 10$ years after eradication in 4 patients, 3 (75\%) of whom had undergone yearly endoscopy before gastric cancer was detected. Two of the 4 lesions (50\%) invaded into the submucosal layer or deeper. Three of the 4 lesions showed lymphovascular invasion.

\section{Discussion}

The malignant potential of UD-GC is thought to be higher than that of D-GC [21]. Therefore, early detection of UD-GC is important. This study focused on clinically significant UD-GC and examined the relationship between UD-GC and HP status (post-eradication, current infection, and no infection). We found that $33.8 \%$ of all lesions in the HP-uninfected group were UD-GC, whereas only $4.5 \%$ of those in the post-eradication group and $6.2 \%$ of those in the current infection group were UD-GC. However, all tumors in the UD-U group were intramucosal lesions and not highly malignant. These cases had the following features: young age, no atrophy in the background mucosa, discolored tone, relatively small tumor size, predominantly pure signet ring cell carcinoma, intramucosal lesions, and no lymphovascular invasion. It has been reported that UD-GC without HP infection is less malignant than UD-GC with current HP infection [9, 10]. The clinicopathological features of UD-GC in patients with eradicated HP and those who were currently infected might be similar in the present study. The clinicopathological features of UD-E and UD-I are similar, including tumor size, microscopic type, color, depth, and the rate of vascular invasion, and the statistical findings are similar compared with UD-U.

Our study differs from previous reports in that we also examined the clinicopathological features of UD-GC that developed after eradication of HP. These cilinicopathological features were similar between the UD-E group and the UD-I group and showed more malignant characteristics than the UD-U group, indicating the possibility that HP infection has a role in promoting gastric cancer.

Fukase et al. reported that eradication of HP reduced the incidence of cancer to about one third [22], and there have also been studies in which eradication did not completely eliminate the risk of cancer even after a long period of time [23-26].

Alarmingly, three of four patients in whom UD-E was detected $\geq 5$ years after eradication had undergone yearly follow-up endoscopy. Although this finding alone does not provide sufficient evidence to conclude that 
yearly follow-up endoscopy after eradication is unnecessary, endoscopists should be aware that some patients who undergo regular endoscopy may still develop cancer that is not curable by endoscopic resection.

The limitations of this study include its single-center setting and lack of surgically treated cases. Furthermore, at less than 100, the number of cases was small. Given the low frequency of UD-GC, particularly UD-E, a multicenter study is needed to accumulate and examine more cases in the future.

\section{Conclusion}

The clinicopathological characteristics of UD-GC were similar between HP-infected patients and HP-eradicated patients. UD-GC was curable by endoscopic resection in all patients without $\mathrm{HP}$ infection.

\section{Abbreviations}

HP: Helicobacter pylori; D-GC: Differentiated-type gastric cancer; UD-GC: Undifferentiated-type gastric cancer; UD-E: Undifferentiated gastric cancer after HP eradication; UD-I: Undifferentiated gastric cancer current HP infection; UD-U: Undifferentiated gastric cancer HP uninfected.

\section{Acknowledgements}

Not applicable.

\section{Authors' contributions}

MT designed of the study, analyzed the data, and wrote the manuscript. SH conceived the study and revised the manuscript critically. DK, KN, YO, TO, JH, YS, YM, ND, HO, SY, and AM collected cases and data about Helicobacter pylori infection status. All authors have read and approved the manuscript.

\section{Funding}

This research received no specific grant from any funding agency in the public, commercial, or not-for-profit sectors.

\section{Availability of data and materials}

The datasets generated during and/or analyzed during the current study are available from the corresponding author on reasonable request. We do not wish to share data because it is identifying or confidential patient data.

\section{Declarations}

Ethics approval and consent to participate

All procedures followed were in accordance with the ethical standards of the responsible committee on human experimentation (institutional and national) and with the Helsinki Declaration of 1964 and later versions. Ethical approval was waived by the local ethics committee of our institution, Toranomon Hospital, approval number 1173, in view of the retrospective nature of the study and all the procedures being performed were part of the routine care. Informed consent was not required in view of the retrospective nature of the study.

\section{Consent for publication}

Not applicable.

\section{Competing interests}

The authors declare that they have no conflict of interest.

Received: 12 July 2021 Accepted: 17 November 2021

Published online: 06 January 2022

\section{References}

1. Correa P. Helicobacter pylori and gastric carcinogenesis. Am J Surg Pathology. 1995;19(Suppl 1):S37-43.

2. IARC Working Group on the Evaluation of Carcinogenic Risks to Humans. IARC monographs on the evaluation of carcinogenic risks to humans. Volume 61. Schistosomes, liver flukes and Helicobacter pylori. International Agency for Research on Cancer, Lyon, France. 1994, pp. 1-241.

3. Parsonnet J, Friedman G, Vandersteen D, Chang Y, Vogelman J, Orentreich $\mathrm{N}$, Sibley RK. Helicobacter pylori infection and the risk of gastric carcinoma. N Engl J Med. 1991;325:1127-31.

4. Kato S, Matsukura N, Tsukada K, Matsuda N, Mizoshita T, Tsukamoto T, Tatematsu M, Sugisaki Y, Naito Z, Tajiri T. Helicobacter pylori-infection negative gastric cancer in Japanese hospital patients: incidence and pathological characteristics. Cancer Sci. 2007;98:790-4.

5. Matsuo T, Ito M, Takata S, Tanaka S, Yoshihara M, Chayama K. Low prevalence of Helicobacter pylori-negative gastric cancer among Japanese. Helicobacter. 2011;16:415-9.

6. Ono S, Kato M, Suzuki M, Ishigaki S, Takahashi M, Haneda M, Mabe K, Shimizu Y. Frequency of Helicobacter pylori-negative gastric cancer and gastric mucosal atrophy in a Japanese endoscopic submucosal dissection series including histological, endoscopic and serological atrophy. Digestion. 2012;86:59-65.

7. Sato C, Hirasawa K, Tateishi Y, Ozeki Y, Sawada A, Ikeda R, Fukuchi T, Nishio M, Kobayashi R, Makazu M, Kaneko H, Inayama Y, Maeda S. Clinicopathological features of early gastric cancers arising in Helicobacter pylori uninfected patients. World J Gastroenterol. 2020;26:2618-31.

8. Yamada A, Kaise M, Inoshita N, Toba T, Nomura K, Kuribayashi Y, Yamashita S, Furuhata T, Kikuchi D, Matsui A, Mitani T, Ogawa O, lizuka T, Hoteya S. Characterization of Helicobacter pylori-naïve early gastric cancers. Digestion. 2018;98:127-34.

9. Yamamoto Y, Fujisaki J, Omae M, Hirasawa T, Igarashi M. Helicobacter pylori-negative gastric cancer: characteristics and endoscopic findings. Dig Endosc. 2015;27:551-61.

10. Yoon H, Kim N, Lee HS, Shin CM, Park YS, Lee DH, Jung HC, Song IS. Helicobacter pylori-negative gastric cancer in South Korea: incidence and clinicopathologic characteristics. Helicobacter. 2011;16:382-8.

11. Hori K, Watari J, Yamasaki T, Kondo T, Toyoshima F, Sakurai J, Ikehara H, Tomita T, Oshima T, Fukui H, Nakamura S, Miwa H. Morphological characteristics of early gastric neoplasms detected after Helicobacer pylori eradication. Dig Dis Sci. 2016;61:1641-51.

12. Ito M, Tanaka S, Takata S, Oka S, Imagawa S, Ueda H, Egi Y, Kitadai Y, Yasui W, Yoshihara M, Haruma K, Chayama K. Morphological changes in human gastric tumours after eradication therapy of Helicobacter pylori in a short-term follow-up. Aliment Pharmacol Ther. 2005;21:559-66.

13. Yamamoto K, Kato M, Takahashi M, Haneda M, Shinada K, Nishida U, Yoshida T, Sonoda N, Ono S, Nakagawa M, Mori Y, Nakagawa S, Mabe K, Shimizu Y, Moriya J, Kubota K, Matsuno Y, Shimoda T, Watanabe H, Asaka M. Clinicopathological analysis of early-stage gastric cancers detected after successful eradication of Helicobacter pylori. Helicobacter. 2011;16:210-6.

14. Kobayashi M, Hashimoto S, Nishikura K, Mizuno K, Takeuchi M, Sato Y, Ajioka Y, Aoyagi Y. Magnifying narrow-band imaging of surface maturation in early differentiated-type gastric cancers after Helicobacter pylori eradication. J Gastroenterol. 2013;48:1332-42.

15. Horiuchi Y, Fujisaki J, Yamamoto N, Shimizu T, Miyamoto Y, Tomida H, Taniguchi C, Morishige K, Omae M, Ishiyama A, Yoshio T, Hirasawa T, Yamamoto Y, Tsuchida T, Igarashi M, Nakajima T, Takahashi H. Biological behavior of the intramucosal Helicobacter pylori-negative undifferentiated-type early gastric cancer: comparison with Helicobacter pyloripositive early gastric cancer. Gastric Cancer. 2016;19:160-5.

16. Ono H, Yao K, Fujishiro M, Oda I, Uedo F, Futamura S, Yahagi N, liishi H, Oka M, Ajioka Y, Fujimoto K. Guidelines for endoscopic submucosal dissection and endoscopic mucosal resection for early gastric cancer. Gastroenterol Endosc. 2020:62:273-90 ((in Japanese)).

17. Haruma K, Kato M, Inoue K, Murakami K, Kamada T. Kyoto classification of gastritis. Tokyo: Nihon Medical Center; 2017. ((in Japanese)).

18. Kimura K, Takemoto T. An endoscopic recognition of the atrophic border and its significance in chronic gastritis. Endoscopy. 1969;1:87-97.

19. Yagi K, Aruga Y, Nakamura A, Sekine A. Regular arrangement of collecting venules (RAC): a characteristic endoscopic feature of Helicobacter 
pylori-negative normal stomach and its relationship with esophagogastric adenocarcinoma. J Gastroenterol. 2005;40:443-52.

20. Japanese Gastric Cancer Association. Japanese classification of gastric carcinoma: 3rd English edition. Gastric Cancer.2011;14:101-112.

21. Hirasawa T, Gotoda T, Miyata S, Kato Y, Shimoda T, Taniguchi H, Fujisaki J, Sano T, Yamaguchi T. Incidence of lymph node metastasis and the feasibility of endoscopic resection for undifferentiated-type early gastric cancer. Gastric Cancer. 2009;12:148-52.

22. Fukase K, Kato M, Kikuchi S, Inoue K, Uemura N, Okamoto S, Terao S, Amagai K, Hayashi S, Asaka M; Japan Gast Study Group. Effect of eradication of Helicobacter pylori on incidence of metachronous gastric carcinoma after endoscopic resection of early gastric cancer: an open-label, randomised controlled trial. Lancet. 2008;372:392-397.

23. Take S, Mizuno M, Ishiki K, Nagahara K, Yoshida T, Yokota K, Oguma K. Baseline gastric mucosal atrophy is a risk factor associated with the development of gastric cancer after Helicobacter pylori eradication therapy in patients with peptic ulcer diseases. J Gastroenterol. 2007;42(Suppl 17):21-7.

24. Take S, Mizuno M, Ishiki K, Yoshida T, Ohara N, Yokota K, Oguma K, Okada $\mathrm{H}$, Yamamoto $\mathrm{K}$. The long-term risk of gastric cancer after the successful eradication of Helicobacter pylori. J Gastroenterol. 2011;46:318-24.

25. Take S, Mizuno M, Ishiki K, Hamada F, Yoshida T, Yokota K, Okada H, Yamamoto K. Seventeen-year effects of eradicating Helicobacter pylori on the prevention of gastric cancer in patients with peptic ulcer; a prospective cohort study. J Gastroenterol. 2015;50:638-44.

26. Take S, Mizuno M, Ishiki K, Kusumoto C, Imada T, Hamada F, Yoshida T, Yokota K, Mitsuhashi T, Okada H. Risk of gastric cancer in the second decade of follow-up after Helicobacter pylori eradication. J Gastroenterol. 2020;55:281-8.

\section{Publisher's Note}

Springer Nature remains neutral with regard to jurisdictional claims in published maps and institutional affiliations.

- fast, convenient online submission

- thorough peer review by experienced researchers in your field

- rapid publication on acceptance

- support for research data, including large and complex data types

- gold Open Access which fosters wider collaboration and increased citations

- maximum visibility for your research: over $100 \mathrm{M}$ website views per year

At BMC, research is always in progress.

Learn more biomedcentral.com/submissions 